\title{
Article \\ Applications of Borel-Type Distributions Series to a Class of Janowski-Type Analytic Functions
}

\author{
Bakhtiar Ahmad $^{1}$ (D), Muhammad Ghaffar Khan ${ }^{2}$ (D) and Luminiţa-Ioana Cotîrlă ${ }^{3, *(D)}$ \\ 1 Government Degree College Mardan, Mardan 23200, Pakistan; pirbakhtiarbacha@gmail.com \\ 2 Institute of Numerical Sciences, Kohat University of Science and Technology Kohat, Kohat 26000, Pakistan; \\ ghaffarkhan020@gmail.com \\ 3 Department of Mathematics, Technical University of Cluj-Napoca, 400114 Cluj-Napoca, Romania \\ * Correspondence: Luminita.Cotirla@math.utcluj.ro
}

check for

check for
updates

Citation: Ahmad, B.; Khan, M.G.; Cotîrlă, L.-I. Applications of Borel-Type Distributions Series to a Class of Janowski-Type Analytic Functions. Symmetry 2022, 14, 322. https://doi.org/10.3390/ sym 14020322

Academic Editors: Şahsene Altınkaya and Alexander Zaslavski

Received: 21 December 2021

Accepted: 1 February 2022

Published: 4 February 2022

Publisher's Note: MDPI stays neutral with regard to jurisdictional claims in published maps and institutional affiliations.

Copyright: (C) 2022 by the authors. Licensee MDPI, Basel, Switzerland. This article is an open access article distributed under the terms and conditions of the Creative Commons Attribution (CC BY) license (https:// creativecommons.org/licenses/by/ $4.0 /)$.

\begin{abstract}
The main purpose of this article is to introduce the new subclass of analytic functions whose coefficients are Borel distributions in the Janowski domain. Further, we investigate some useful number of properties such as Fekete-Szeg ö inequality, necessary and sufficient condition, growth and distortion approximations, convex linear combination, arithmetic mean, radii of close-to-convexity and starlikeness and partial sums, followed by some extremal functions for this defined class. The symmetry properties and other properties of the subclass of functions introduced in this paper can be studied as future research directions.
\end{abstract}

Keywords: analytic functions; subordinations; borel distribution series; subordinations; radii problems

\section{Introduction and Motivation}

Let $\mathcal{A}_{0}$ represent the collections of analytic functions $f$ inside open unit disc $\mathbb{D}=$ $\{\xi \in \mathbb{C}:|\xi|<1\}$ with normalized form

$$
f(\xi)=\xi+\sum_{n=2}^{\infty} a_{n} \xi^{n}, \xi \in \mathbb{D} .
$$

Moreover, as indicated by $\mathcal{S}$, a subclass of $\mathcal{A}_{0}$ consists of all functions that are univalent inside open unit disc $\mathbb{D}$. A set $\Lambda \subset \mathbb{C}$ is called starlike with respect to origin if, for any point in set $\Lambda$, the line segment joining origin to that point lies inside $\Lambda$.

A function $f \in \mathcal{A}_{0}$ and maps $\mathbb{D}$ to a starlike region is called starlike functions and the class of such functions is denoted by $\mathcal{S}^{*}$. Analytically, a function $f \in \mathcal{A}_{0}$ is called starlike function if

$$
\Re\left(\frac{\xi f^{\prime}(\xi)}{f(\xi)}\right)>0,(\xi \in \mathbb{D}) .
$$

A set $\Lambda \subset \mathbb{C}$ is called convex if, for any two points, $\xi_{1}$ and $\xi_{2}$ in set $\Lambda$ if the line segment joining these two points lies inside $\Lambda$. A function $f \in \mathcal{A}_{0}$ and maps $\mathbb{D}$ to a convex shaped domain is called a convex function and the class of such functions is denoted by $\mathcal{C}$. Analytically, a function $f \in \mathcal{A}_{0}$ is called a convex function if

$$
\Re\left(1+\frac{\xi f^{\prime \prime}(\xi)}{f^{\prime}(\xi)}\right)>0,(\xi \in \mathbb{D}) .
$$

Since the early twentieth century, many mathematicians have been interested in different problems involving the coefficients of functions $f$ in a given subclass of $\mathcal{A}_{0}$. The most important and inspiring problem, known as the Bieberbach conjecture, which was solved by de Branges in 1984, 70 years after its formulation. Over the years, many 
interesting tasks connected with these coefficients appeared. The Fekete-Szegö functional $\left|a_{3}-a_{2}^{2}\right|$ is also one an important finding for the coefficients of the functions $f$. This functional is further generalized as $\left|a_{3}-\mu a_{2}^{2}\right|$ for some $\mu$ real as well as complex. Fekete and Szegö gave sharp estimates of $\left|a_{3}-\mu a_{2}^{2}\right|$ for a real $\mu$ and $f \in \mathcal{S}$, the class of univalent functions.

In Geometric Function Theory, the elementary distributions such as the Pascal, Poisson, logarithmic, binomial and beta negative binomial, have been partially studied from a theoretical point of view. For a detailed study, we refer the readers to [1-5].

Wanas and Khuttar [6], recently introduced the power series whose coefficients are probabilities of the Borel distribution:

$$
S(r, \xi)=\xi+\sum_{n=2}^{\infty} \frac{(r(n-1))^{n-2} e^{-r(n-1)}}{(n-1) !} \xi^{n},(\xi \in \mathbb{D} ; 0<r \leq 1) .
$$

From a well-known ratio test, the above series is convergent with the domain of convergence of the entire complex plane.

Utilizing the above, the authors in [6] defined the linear operator $Q_{r}: \mathcal{A}_{0} \rightarrow \mathcal{A}_{0}$ as:

$$
\begin{aligned}
Q_{r} f(\xi) & =f(\xi) * S(r, \xi) \\
& =\xi+\sum_{n=2}^{\infty} \frac{(r(n-1))^{n-2} e^{-r(n-1)}}{(n-1) !} a_{n} \xi^{n},
\end{aligned}
$$

where the symbol $*$ specifies the Hadamard product (convolution) of two series.

Moreover, for two functions $f$ and $g$ analytic in $\mathbb{D}$, we say that the function $f$ is subordinate to the function $g$ and write this as

$$
f \prec g \quad \text { or } \quad f(\xi) \prec g(\xi),
$$

if there a Schwarz function $w$ exists, which is analytic in $\mathbb{D}$ with

$$
w(0)=0 \quad \text { and } \quad|w(\xi)|<1,
$$

such that

$$
f(\xi)=g(w(\xi)) .
$$

Furthermore, if the function $g$ is univalent in $\mathbb{D}$, then it follows that:

$$
f(\xi) \prec g(\xi) \quad(\xi \in \mathbb{D}) \Rightarrow f(0)=g(0) \text { and } f(\mathbb{D}) \subset g(\mathbb{D}) .
$$

The study of operators plays an important role in Geometric Function Theory. Many differential and integral operators can be written in terms of the convolution of certain analytic functions. It is observed that this formalism makes further mathematical exploration easier, and also improves the understanding of the geometric and symmetric properties of such operators. The importance of convolution in the theory of operators may easily be understood from the work in [7-10]. Furthermore, probability is not just about flipping coins and counting cards in a disc; it is used in a wide range of real-life areas, from insurance to meteorology and politics to economics forecasting. For more applications, we refer the reader to [11-15].

Motivated from all the above discussions and work from Khan et al. [16], in which they introduced a class of analytic functions with Mittag-Leffler type Poisson distribution in the Janowski domain, analytic functions with Mittag-Leffler type Borel distribution [17], and the work in the articles [18,19], we now introduce a new class of analytic functions with the help of operator (2), as follows:

$$
\mathcal{S}_{\mathcal{B}}^{*}(A, B)=\left\{f \in \mathcal{A}_{0}: \frac{\xi Q_{r}^{\prime} f(\xi)}{Q_{r} f(\xi)} \prec \frac{1+A \xi}{1+B \xi}\right\} \quad(\xi \in \mathbb{D}),
$$


where $-1 \leq B<A \leq 1$.

To find the Fekete-Szegö problem, we need the following Lemma.

Lemma 1. In $[20,21]$ Let

$$
h(\xi)=1+p_{1} \xi+p_{2} \xi^{2}+\ldots
$$

is in the class $\mathcal{P}$ of functions of the positive real part in $\mathbb{D}$, then

$$
\left|p_{n}\right| \leq 1, n \geq 1
$$

and, for any complex number $v$

$$
\left|p_{2}-v p_{1}^{2}\right| \leq 2 \max \{1,|1-2 v|\} .
$$

In particular, if $v$ is a real parameter, then

$$
\left|p_{2}-v p_{1}^{2}\right| \leq \begin{cases}-4 v+2 & (v \leq 0) \\ 2 & (0 \leq v \leq 1) \\ 4 v-2 & (v \geq 1)\end{cases}
$$

When $v<0$ or $v>1$, equality holds true in (6) if and only if

$$
h(\xi)=\frac{1+\xi}{1-\xi}
$$

or one of its rotations. If $0<v<1$, then equality holds true in (6) if and only if

$$
h(\xi)=\frac{1+\xi^{2}}{1-\xi^{2}}
$$

or one of its rotations. If $v=0$, equality holds true in (6) if and only if

$$
h(\xi)=\left(\frac{1+\rho}{2}\right) \frac{1+\xi}{1-\xi}+\left(\frac{1-\rho}{2}\right) \frac{1-\xi}{1+\xi} \quad(0 \leq \rho \leq 1)
$$

or one of its rotations. If $v=1$, then the equality in (6) holds true if $h(\xi)$ is a reciprocal of one of the functions, such that the equality holds true in the case when $v=0$.

\section{Main Results}

In the present paper, we evaluate Fekete-Szegö inequality, necessary and sufficient conditions, growth and distortion bounds, radii of starlikeness and convexity, radii of close-to-convexity and partial sums results for the newly defined class.

Theorem 1. Let $f \in \mathcal{A}_{0}$ be assigned to the class $\mathcal{S}_{\mathcal{B}}^{*}(A, B)$. Then,

$$
\begin{aligned}
& \left|a_{2}\right| \leq \frac{A-B}{\Lambda_{2}} \\
& \left|a_{3}\right| \leq \frac{A-B}{\Lambda_{3}} \max \{1,|2 B-A|\} .
\end{aligned}
$$

Additionally, for a complex number $\lambda$,

$$
\left|a_{3}-\mu a_{2}^{2}\right| \leq \frac{A-B}{\Lambda_{3}} \max \{1,|\Psi(\lambda, A, B)-1|\},
$$


where

$$
\Psi(\lambda, A, B)=\frac{\Lambda_{3}}{\Lambda_{2}^{2}}\left[2 \Lambda_{2}^{2}(1+2 B-A)+\lambda(A-B)\right]
$$

and

$$
\Lambda_{n}=(r(n-1))^{n-2} e^{-r(n-1)} .
$$

Furthermore, for a real parameter $\lambda$,

$$
\left|a_{3}-\lambda a_{2}^{2}\right| \leq \begin{cases}\frac{A-B}{\Lambda_{3}}(1-\Psi(\lambda, A, B)) & \left(\lambda<\sigma_{1}\right) \\ \frac{A-B}{\Lambda_{3}} & \left(\sigma_{1} \leq \lambda \leq \sigma_{2}\right) \\ \frac{A-B}{\Lambda_{3}}(\Psi(\lambda, A, B)-1) & \left(\lambda>\sigma_{2}\right)\end{cases}
$$

where

$$
\begin{gathered}
\Psi(\lambda, A, B)=\frac{\Lambda_{3}}{\Lambda_{2}^{2}}\left[2 \Lambda_{2}^{2}(1+2 B-A)+\lambda(A-B)\right] \\
\sigma_{1}=-\frac{2 \Lambda_{2}^{2}(1+2 B-A)}{(A-B)}
\end{gathered}
$$

and

$$
\sigma_{2}=\frac{2 \Lambda_{2}^{2}\left(1-\Lambda_{3}(1+2 B-A)\right)}{\Lambda_{3}(A-B)}
$$

Proof. We begin by showing that the inequalities (7)-(10) hold true for $f \in \mathcal{S}_{\mathcal{B}}^{*}(A, B)$. Since $f \in \mathcal{S}_{\mathcal{B}}^{*}(A, B)$, therefore, we have the following subordination:

$$
\frac{\xi Q_{r}^{\prime} f(\xi)}{Q_{r} f(\xi)} \prec \frac{1+A \xi}{1+B \xi}
$$

The above subordination can also be written as:

$$
\frac{\xi Q_{r}^{\prime} f(\xi)}{Q_{r} f(\xi)}=\frac{1+A w(\xi)}{1+B w(\xi)}=G(w(\xi)), \quad(-1 \leq B<A \leq 1)
$$

Now, $w(\xi)$ can be written as follows:

$$
w(\xi)=\frac{1-h(\xi)}{1+h(\xi)}=\frac{p_{1} \xi+p_{2} \xi^{2}+p_{3} \xi^{3}+\cdots}{2+p_{1} \xi+p_{2} \xi^{2}+p_{3} \xi^{3}+\cdots} .
$$

Now,

$$
G(w(\xi))=1+\frac{1}{2}(A-B) p_{1} \xi+\frac{1}{4}\left(2(A-B) p_{2}-(1+B) p_{1}^{2}\right) \xi^{2}+\cdots .
$$

Additionally,

$$
\frac{\xi Q_{r}^{\prime} f(\xi)}{Q_{r} f(\xi)}=1+\Lambda_{2} a_{2} \xi+\left(\Lambda_{3} a_{3}-\Lambda_{2}^{2} a_{2}^{2}\right) \xi^{2}+\ldots
$$

After comparing (13) and (14), we obtain

$$
\begin{aligned}
& a_{2}=\frac{A-B}{2 \Lambda_{2}} p_{1} \\
& a_{3}=\frac{A-B}{2 \Lambda_{3}}\left(p_{2}-\frac{1+2 B-A}{2} p_{1}^{2}\right) .
\end{aligned}
$$


applying (4) to (15) and (5) to (16), we obtain

$$
\begin{aligned}
& \left|a_{2}\right| \leq \frac{A-B}{\Lambda_{2}} \\
& \left|a_{3}\right| \leq \frac{A-B}{\Lambda_{3}} \max \{1,|2 B-A|\} .
\end{aligned}
$$

Additionally, from (15) and (16), we obtain

$$
\left|a_{3}-\lambda a_{2}^{2}\right|=\frac{A-B}{2 \Lambda_{3}}\left|p_{2}-\Psi(\lambda, A, B) p_{1}^{2}\right|,
$$

where $\Psi(\lambda, A, B)$ is defined in (11). Applying (5) to above (19) we obtain the required results. Additionally, for real $\lambda$ applying (6) to above (19), we obtain the required results.

Theorem 2. Let $f \in \mathcal{A}_{0}$ is assigned to the class $\mathcal{S}_{\mathcal{B}}^{*}(A, B)$ if fulfils the inequlity

$$
\sum_{n=2}^{\infty}\left(\frac{(1-B) n+(A-1)}{(n-1) !}\right) \Lambda_{n}\left|a_{n}\right| \leq A-B,
$$

where

$$
\Lambda_{n}=(r(n-1))^{n-2} e^{-r(n-1)} .
$$

Proof. Let $f \in \mathcal{S}_{\mathcal{B}}^{*}(A, B)$. Then, one can put (3) in the form of Schwarz function $w(\xi)$, as

$$
\frac{\xi Q_{r}^{\prime} f(\xi)}{Q_{r} f(\xi)}=\frac{1+A w(\xi)}{1+B w(\xi)}(\xi \in \mathbb{D}) .
$$

Alternately, and equivalently,

$$
\left|\frac{\xi Q_{r}^{\prime} f(\xi)-Q_{r} f(\xi)}{A Q_{r} f(\xi)-B \xi Q_{r}^{\prime} f(\xi)}\right|<1 .
$$

Consider the following, and put $|\xi|=t$

$$
\begin{aligned}
& \left|\frac{\xi Q_{r}^{\prime} f(\xi)-Q_{r} f(\xi)}{A Q_{r} f(\xi)-B \xi Q_{r}^{\prime} f(\xi)}\right| \\
= & \left|\frac{\sum_{n=2}^{\infty} \frac{(n-1)}{(n-1) !}(r(n-1))^{n-2} e^{-r(n-1)} a_{n} \xi^{n}}{(A-B) \xi+\sum_{n=2}^{\infty} \frac{(A-B n)}{(n-1) !}(r(n-1))^{n-2} e^{-r(n-1)} a_{n} \xi^{n}}\right| \\
\leq & \frac{\sum_{n=2}^{\infty} \frac{(n-1)}{(n-1) !}(r(n-1))^{n-2} e^{-r(n-1)}\left|a_{n}\right| t^{n-1}}{(A-B)-\sum_{n=2}^{\infty} \frac{(A-B n)}{(n-1) !}(r(n-1))^{n-2} e^{-r(n-1)}\left|a_{n}\right| t^{n-1}}<1,
\end{aligned}
$$

By letting $t \rightarrow 1$ and performing a simple computation, we obtain the desired inequlity (20).

Example 1. For the function

$$
f(\xi)=\xi+\sum_{n=2}^{\infty} \frac{(n-1) !(A-B)}{[(1-B) n+(A-1)] \Lambda_{n}} t_{n} \xi^{n}, \xi \in \mathbb{D},
$$


such that $\sum_{n=2}^{\infty} t_{n}=1$, we have

$$
\begin{aligned}
& \sum_{n=2}^{\infty} \frac{(1-B) n+(A-1)}{(n-1) !} \Lambda_{n}\left|a_{n}\right| \\
= & \sum_{n=2}^{\infty} \frac{[(1-B) n+(A-1)] \Lambda_{n}}{(n-1) !}\left(\frac{(n-1) !(A-B)}{[(1-B) n+(A-1)] \Lambda_{n}} t_{n}\right) \\
= & (A-B) \sum_{n=2}^{\infty} t_{n}=(A-B) .
\end{aligned}
$$

Thus, $f(\xi) \in \mathcal{S}_{\mathcal{B}}^{*}(A, B)$, and the approximation (20) is sharp.

Corollary 1. Let $f \in \mathcal{S}_{\mathcal{B}}^{*}(A, B)$ and be of the form (1). Then,

$$
\left|a_{n}\right| \leq \frac{(n-1) !(A-B)}{[(1-B) n+(A-1)] \Lambda_{n}}, \text { for } n \geq 2,
$$

where $\Lambda_{n}$ is defined in (21). In addition, the approximation is sharp for function

$$
f(\xi)=\xi-\frac{(n-1) !(A-B)}{[(1-B) n+(A-1)] \Lambda_{n}} \xi^{n}, \text { for } n \geq 2 .
$$

Proof. The proof is quite straightforward, and left for the reader.

Theorem 3. A function $f \in \mathcal{A}_{0}$ assigned to the class $\mathcal{S}_{\mathcal{B}}^{*}(A, B)$. Then

$$
r-\frac{(A-B)}{(1+2 B-A) \Lambda_{2}} r^{2} \leq|f(\xi)| \leq r+\frac{(A-B)}{(1+2 B-A) \Lambda_{2}} r^{2} .
$$

The approximation is sharp for functions defined as follows:

$$
f(\xi)=\xi-\frac{(A-B)}{(1+2 B-A) \Lambda_{2}} \xi^{2}
$$

Proof. Consider

$$
\begin{aligned}
|f(\xi)| & =\left|\xi+\sum_{n=2}^{\infty} a_{n} \xi^{n}\right| \\
& \leq|\xi|+\sum_{n=2}^{\infty} a_{n}|\xi|^{n} \\
& =r+\sum_{n=2}^{\infty} a_{n}|r|^{n}
\end{aligned}
$$

since, for $|\xi|=r<1$, we have $r^{n}<r^{2}$ for $n \geq 2$ and

$$
|f(\xi)| \leq r+r^{2} \sum_{n=2}^{\infty}\left|a_{n}\right|
$$

Comparably,

$$
|f(\xi)| \geq r-r^{2} \sum_{n=2}^{\infty}\left|a_{n}\right| .
$$

Now, from (20), implying that

$$
\sum_{n=2}^{\infty} \frac{(1-B) n+(A-1)}{(n-1) !} \Lambda_{n}\left|a_{n}\right| \leq A-B
$$


However,

$$
\begin{aligned}
& \sum_{n=2}^{\infty}(1+2 B-A) \Lambda_{2}\left|a_{n}\right| \\
\leq & \sum_{n=2}^{\infty} \frac{(1-B) n+(A-1)}{(n-1) !} \Lambda_{n}\left|a_{n}\right| \leq A-B,
\end{aligned}
$$

which gives

$$
\sum_{n=2}^{\infty} a_{n} \leq \frac{(A-B)}{(1+2 B-A) \Lambda_{2}} .
$$

Applying this inequality to the above inequalities (27) and (28), we obtain the desired result.

Theorem 4. A function $f \in \mathcal{A}_{0}$ assigned to the class $\mathcal{S}_{\mathcal{B}}^{*}(A, B)$. Then,

$$
1-\frac{2(A-B)}{(1+2 B-A) \Lambda_{2}} r \leq\left|f^{\prime}(\xi)\right| \leq 1+\frac{2(A-B)}{(1+2 B-A) \Lambda_{2}} r .
$$

The result is sharp for the extreme function defined in (26).

Proof. The proof is quite similar to Theorem 3, and therefore it was omitted.

Theorem 5. Let $f_{i} \in \mathcal{S}_{\mathcal{B}}^{*}(A, B)$ and have of the from

$$
f_{i}(\xi)=\xi+\sum_{n=2}^{\infty} a_{i, n} \xi^{n}, \text { for } i=1,2,3, \ldots, k
$$

Then, $H \in \mathcal{S}_{\mathcal{B}}^{*}(A, B)$, where

$$
H(\xi)=\sum_{i=1}^{k} c_{i} f_{i}(\xi) \text { with } \sum_{i=1}^{k} c_{i}=1
$$

Proof. In light of Theorem 2, we can write

$$
\sum_{n=2}^{\infty} \frac{(1-B) n+(A-1)}{(n-1) !} \Lambda_{n}\left|a_{n}\right| \leq A-B .
$$

Additionally,

$$
\begin{aligned}
H(\xi) & =\sum_{i=1}^{k} c_{i}\left(\xi+\sum_{n=2}^{\infty} a_{i, n} \xi^{n}\right) \\
& =\xi+\sum_{n=2}^{\infty}\left(\sum_{i=1}^{k} c_{i} a_{i, n}\right) \xi^{n}
\end{aligned}
$$

therefore,

$$
\begin{aligned}
& \sum_{n=2}^{\infty} \frac{(1-B) n+(A-1)}{(n-1) !} \Lambda_{n}\left|\sum_{i=1}^{k} c_{i} a_{i, n}\right| \\
= & \sum_{i=1}^{k}\left[\sum_{n=2}^{\infty} \frac{(1-B) n+(A-1)}{(n-1) !} \Lambda_{n}\left|a_{i, n}\right|\right] c_{i} \\
\leq & \sum_{i=1}^{k}(A-B) c_{i}=(A-B) \sum_{i=1}^{k} c_{i}=A-B,
\end{aligned}
$$


thus $H(\xi) \in \mathcal{S}_{\mathcal{B}}^{*}(A, B)$.

Theorem 6. Let $f_{i} \in \mathcal{S}_{\mathcal{B}}^{*}(A, B)$, for $i=1,2, \ldots, j$. Then, the arithmetic mean $\mathcal{G}$ of $f_{i}$ is given by

$$
\mathcal{G}(\xi)=\frac{1}{j} \sum_{n=1}^{j} f_{i}(\xi)
$$

and also belongs to class $\mathcal{S}_{\mathcal{B}}^{*}(A, B)$.

Proof. From (32), we can write

$$
\begin{aligned}
\mathcal{G}(\xi) & =\frac{1}{j} \sum_{n=1}^{j} f_{i}(\xi) \\
& =\frac{1}{j} \sum_{n=1}^{j}\left(\xi+\sum_{n=2}^{\infty} a_{j, n} \xi^{n}\right) \\
& =\xi+\sum_{n=2}^{\infty}\left(\frac{1}{j} \sum_{n=1}^{j} a_{j, n}\right) \xi^{n}
\end{aligned}
$$

to show that $\mathcal{G}(\xi)$ belongs to $\mathcal{S}_{\mathcal{B}}^{*}(A, B)$, it is enough to show that

$$
\sum_{n=2}^{\infty} \frac{(1-B) n+(A-1)}{(n-1) !} \Lambda_{n}\left(\frac{1}{j} \sum_{n=1}^{j}\left|a_{j, n}\right|\right) \leq A-B .
$$

Consider

$$
\begin{aligned}
& \sum_{n=2}^{\infty} \frac{(1-B) n+(A-1)}{(n-1) !} \Lambda_{n}\left(\frac{1}{j} \sum_{n=1}^{j}\left|a_{j, n}\right|\right) \\
= & \frac{1}{j} \sum_{n=1}^{j}\left(\sum_{n=2}^{\infty} \frac{(1-B) n+(A-1)}{(n-1) !} \Lambda_{n}\left|a_{j, n}\right|\right) \\
\leq & \frac{1}{j} \sum_{n=1}^{j}(A-B)=(A-B),
\end{aligned}
$$

this shows that $\mathcal{G}(\xi)$ belongs to $\mathcal{S}_{\mathcal{B}}^{*}(A, B)$.

Theorem 7. Let $f \in \mathcal{S}_{\mathcal{B}}^{*}(A, B)$, then $f$ is in a class of starlike functions of the order $\beta(0 \leq \beta<1)$ for $|\xi|<r^{*}$, where

$$
r^{*}=\inf _{n \geq 2}\left(\frac{(1-\beta)[(1-B) n+(A-1)]}{(n-1) !(n-\beta)} \Lambda_{n}\right)^{\frac{1}{n-1}} .
$$

The result is sharp for the extreme function defined in (24).

Proof. Let $f \in \mathcal{S}_{\mathcal{B}}^{*}(A, B)$. To prove $f$ is in a class of starlike functions of the order $\beta$, it is enough to show that

$$
\left|\frac{\xi f^{\prime}(\xi)}{f(\xi)}-1\right|<1-\beta
$$

Using routine simplifications, we obtain

$$
\sum_{n=2}^{\infty}\left(\frac{n-\beta}{1-\beta}\right)\left|a_{n}\right||\xi|^{n-1}<1
$$


Since $f \in \mathcal{S}_{\mathcal{B}}^{*}(A, B)$, from (20), we have

$$
\sum_{n=2}^{\infty} \frac{(1-B) n+(A-1)}{(n-1) !} \Lambda_{n}\left|a_{n}\right|<1 .
$$

Inequality (33) will hold true if the following holds true:

$$
\begin{aligned}
& \sum_{n=2}^{\infty}\left(\frac{n-\beta}{1-\beta}\right)\left|a_{n}\right||\xi|^{n-1} \\
< & \sum_{n=2}^{\infty} \frac{(1-B) n+(A-1)}{(n-1) !} \Lambda_{n}\left|a_{n}\right|,
\end{aligned}
$$

which implies that

$$
\begin{aligned}
|\xi|^{n-1} & <\left(\frac{(1-\beta)[(1-B) n+(A-1)]}{(n-1) !(n-\beta)} \Lambda_{n}\right), \\
|\xi| & <\left(\frac{(1-\beta)[(1-B) n+(A-1)]}{(n-1) !(n-\beta)} \Lambda_{n}\right)^{\frac{1}{n-1}},
\end{aligned}
$$

thus, we obtain the required result.

Theorem 8. Let $f \in \mathcal{S}_{\mathcal{B}}^{*}(A, B)$, then $f$ is in a class of close-to-convex functions of the order $\beta$ $(0 \leq \beta<1)$ for $|\xi|<r_{1}^{*}$, where

$$
r_{1}^{*}=\inf _{n \geq 2}\left(\frac{(1-\beta)[(1-B) n+(A-1)]}{n !} \Lambda_{n}\right)^{\frac{1}{n-1}} .
$$

Proof. Let $f \in \mathcal{S}_{\mathcal{B}}^{*}(A, B)$. To prove $f$ is in a class of close-to-convex functions of the order $\beta$, it is enough to show that

$$
\left|f^{\prime}(\xi)-1\right|<1-\beta
$$

Using routine simplifications, we obtain

$$
\sum_{n=2}^{\infty} \frac{n}{1-\beta}\left|a_{n}\right||\xi|^{n-1}<1
$$

Since $f \in \mathcal{S}_{\mathcal{B}}^{*}(A, B)$, from (20), we have

$$
\sum_{n=2}^{\infty} \frac{(1-B) n+(A-1)}{(n-1) !} \Lambda_{n}\left|a_{n}\right|<1 .
$$

Inequality (33) will hold true if the following holds true:

$$
\begin{aligned}
& \sum_{n=2}^{\infty} \frac{n}{1-\beta}\left|a_{n}\right||\xi|^{n-1} \\
< & \sum_{n=2}^{\infty} \frac{(1-B) n+(A-1)}{(n-1) !} \Lambda_{n}\left|a_{n}\right|,
\end{aligned}
$$

which implies that

$$
\begin{aligned}
|\xi|^{n-1} & <\left(\frac{(1-\beta)[(1-B) n+(A-1)]}{n !} \Lambda_{n}\right), \\
|\xi| & <\left(\frac{(1-\beta)[(1-B) n+(A-1)]}{n !} \Lambda_{n}\right)^{\frac{1}{n-1}},
\end{aligned}
$$


Thus, we obtain the required result.

\section{Partial Sums}

In 1997, Silverman [22], examined partial sum results for the class of starlike and convex functions $f$ diven by (1) and established through

$$
\begin{aligned}
& f_{1}(\xi)=\xi \\
& f_{m}(\xi)=\xi+\sum_{n=2}^{m} a_{n} \xi^{n},=2,3,4, \ldots
\end{aligned}
$$

After this, several authors investigated partial sums for different subclasses and, after some recent investigations, we refer authors to $[16,23,24]$.

Theorem 9. If $f$ of the form (1) satisfies condition (20), then

$$
\Re\left(\frac{f(\xi)}{f_{j}(\xi)}\right) \geq 1-\frac{1}{\alpha_{j+1}} \quad(\forall \xi \in \mathbb{D})
$$

and

$$
\Re\left(\frac{f_{j}(\xi)}{f(\xi)}\right) \geq \frac{\alpha_{j+1}}{1+\alpha_{j+1}} \quad(\forall \xi \in \mathbb{D}),
$$

where

$$
\alpha_{j}=\frac{(1-B) n+(A-1)}{(n-1) !(A-B)} \Lambda_{n} .
$$

Proof. To prove the approximation (37), we use:

$$
\begin{aligned}
\alpha_{j+1}\left[\frac{f(\xi)}{f_{j}(\xi)}-\left(1-\frac{1}{\alpha_{j+1}}\right)\right] & =\frac{1+\sum_{n=2}^{j} a_{n} \xi^{n-1}+\alpha_{j+1} \sum_{n=j+1}^{\infty} a_{n} \xi^{n-1}}{1+\sum_{n=2}^{j} a_{n} \xi^{n-1}} \\
& =\frac{1+\psi_{1}(\xi)}{1+\psi_{2}(\xi)} .
\end{aligned}
$$

We now set:

$$
\frac{1+\psi_{1}(\xi)}{1+\psi_{2}(\xi)}=\frac{1+w(\xi)}{1-w(\xi)} .
$$

Then, we find after some worthy simplification, that:

$$
w(\xi)=\frac{\psi_{1}(\xi)-\psi_{2}(\xi)}{2+\psi_{1}(\xi)+\psi_{2}(\xi)} .
$$

Thus, clearly, we find that:

$$
w(\xi)=\frac{\alpha_{j+1} \sum_{n=j+1}^{\infty} a_{n} \xi^{n-1}}{2+2 \sum_{n=2}^{j} a_{n} \xi^{n-1}+\alpha_{j+1} \sum_{n=j+1}^{\infty} a_{n} \xi^{n-1}}
$$


By implementation of the triangle inequalities with $|\xi|<1$, we reached the following inequality:

$$
|w(\xi)| \leq \frac{\alpha_{j+1} \sum_{n=j+1}^{\infty}\left|a_{n}\right|}{2-2 \sum_{n=2}^{j}\left|a_{n}\right|-\alpha_{j+1} \sum_{n=j+1}^{\infty}\left|a_{n}\right|} .
$$

We can now see that:

$$
|w(\xi)| \leq 1
$$

if and only if

$$
2 \alpha_{j+1} \sum_{n=j+1}^{\infty}\left|a_{n}\right| \leq 2-2 \sum_{n=2}^{j}\left|a_{n}\right|
$$

which hints that:

$$
\sum_{n=2}^{j}\left|a_{n}\right|+\alpha_{j+1} \sum_{n=j+1}^{\infty}\left|a_{n}\right| \leq 1
$$

Finally, to prove the inequality in (37), it suffices to show that the left-hand side of (40) is bounded above by the following sum:

$$
\sum_{n=2}^{\infty} \alpha_{n}\left|a_{n}\right|
$$

which is equivalent to

$$
\sum_{n=2}^{j}\left(\alpha_{n}-1\right)\left|a_{n}\right|+\sum_{n=j+1}^{\infty}\left(\alpha_{n}-\alpha_{j+1}\right)\left|a_{n}\right| \geq 0 .
$$

In light of (41), this is evidence that the proof of approximation in (37) is now completed.

Next, in order to prove the inequality (38), we set:

$$
\begin{aligned}
\left(1+\alpha_{j+1}\right)\left(\frac{f_{j}(\xi)}{f(\xi)}-\frac{\alpha_{j+1}}{1+\alpha_{j+1}}\right) & =\frac{1+\sum_{n=2}^{j} a_{n} \xi^{n-1}-\alpha_{j+1} \sum_{n=j+1}^{\infty} a_{n} \xi^{n-1}}{1+\sum_{n=2}^{\infty} a_{n} \xi^{n-1}} \\
& =\frac{1+w(\xi)}{1-w(\xi)}
\end{aligned}
$$

where

$$
|w(\xi)| \leq \frac{\left(1+\alpha_{j+1}\right) \sum_{n=j+1}^{\infty}\left|a_{n}\right|}{2-2 \sum_{n=2}^{j}\left|a_{n}\right|-\left(\alpha_{j+1}-1\right) \sum_{n=j+1}^{\infty}\left|a_{n}\right|} \leq 1 .
$$

This last inequality in (42) is equivalent to

$$
\sum_{n=2}^{j}\left|a_{n}\right|+\alpha_{j+1} \sum_{n=j+1}^{\infty}\left|a_{n}\right| \leq 1 .
$$

Finally, we can see that the left-hand side of the inequality in (43) is bounded above by the following sum:

$$
\sum_{n=2}^{\infty} \alpha_{n}\left|a_{n}\right|
$$


so we have completed the proof of the assertion(38). This completes the proof of Theorem 9.

We next turn to ratios involving derivatives.

Theorem 10. If $f$ of the form (1) satisfies condition (20), then

$$
\Re\left(\frac{f^{\prime}(\xi)}{f_{j}^{\prime}(\xi)}\right) \geq 1-\frac{j+1}{\alpha_{j+1}} \quad(\forall \xi \in \mathbb{D})
$$

and

$$
\Re\left(\frac{f_{j}^{\prime}(\xi)}{f^{\prime}(\xi)}\right) \geq \frac{\alpha_{j+1}}{\alpha_{j+1}+j+1} \quad(\forall \xi \in \mathbb{D}),
$$

where $\alpha_{j}$ is given by (39).

Proof. The proof of Theorem 10 is similar to that of Theorem 9; here, we chose to omit the analogous details.

\section{Conclusions}

In this paper, by making use of the well-known Borel distribution series, a new class of analytic functions was systematically defined. Then, for this newly defined functions class, we studied a number of well-known results, such as the Fekete-Szegö inequalities, the necessary and sufficient conditions, the growth and distortion bounds, the radii of close-to-convexity and starlikeness and partial sums type results. Furthermore, we believe that this study will motivate a number of researchers to extend this idea to meromorphic functions, bi-univalent functions, harmonic functions, $q$-calculus and $(p, q)$-calculus. One may also apply this idea to the use sine domain, cosine domain and petal shaped domain instead of the Janowski domain.

Author Contributions: Conceptualization, B.A., M.G.K. and L.-I.C.; methodology, B.A., M.G.K. and L.-I.C.; software, B.A., M.G.K. and L.-I.C.; validation, B.A., M.G.K. and L.-I.C.; formal analysis, B.A., M.G.K. and L.-I.C.; investigation, B.A., M.G.K. and L.-I.C.; resources, B.A., M.G.K. and L.-I.C.; data curation,B.A., M.G.K. and L.-I.C.; writing-original draft preparation, B.A., M.G.K. and L.I.C.; writing-review and editing, B.A., M.G.K. and L.-I.C.; visualization, B.A., M.G.K. and L.-I.C. supervision, B.A., M.G.K. and L.-I.C.; project administration, B.A., M.G.K. and L.-I.C.; funding acquisition, B.A., M.G.K. and L.-I.C. All authors have read and agreed to the published version of the manuscript.

Funding: This research received no external funding.

Institutional Review Board Statement: Not applicable.

Informed Consent Statement: Not applicable.

Data Availability Statement: Not applicable.

Acknowledgments: The authors would like to thank the referees for their careful reading and helpful comments.

Conflicts of Interest: The authors declare that they have no competing interest.

\section{References}

1. Altinkaya, Ş.; Yalçin, S. Poisson distribution series for certain subclasses of starlike functions with negative coefficients. Ann. Oradea Univ. Math. Fasc. 2017, 24, 5-8.

2. $\quad$ El-Deeb, S.M. ; Bulboaca, T. ; Dziok, J. Pascal distribution series connected with certain subclasses of univalent functions. Kyungpook Math. J. 2019, 59, 301-314.

3. Nazeer, W. ; Mehmood, Q.; Kang, S. M.; Haq, A. U. An application of Bionomial distribution series on certain analytic functions. J. Comput. Anal. Appl. 2019, 26, 11-17.

4. Porwal S.; Kumar, M. A unified study on starlike and convex functions associated with Poisson distribution series. Afr. Mat. 2016, 27, 10-21. [CrossRef] 
5. Wanas, A.K.; Al-Ziadi, N. A. Applications of beta negative binomial distribution series on holomorphic funxtions. Earthline J. Math. Sci. 2021, 6, 271-292. [CrossRef]

6. Wanas, A.K.; Khuttar, J.A. Applications of Borel distribution series on analytic functions. Earthline J. Math. Sci. 2020, 4, 71-82. [CrossRef]

7. Ahmad, B.; Khan, M.G.; Aouf, M.K.; Mashwani, W.K.; Salleh, Z. Tang, H. Applications of a new q-difference operator in Janowski-type meromorphic convex functions. J. Funct. 2021, 2021, 5534357.

8. Khan, B.; Srivastava, H.M.; Arjika, S. A certain q-Ruscheweyh type derivative operator and its applications involving multivalent functions. Adv. Differ. Equ. 2021, 2021, 279. [CrossRef]

9. Shi, L.; Ahmad, B.; Khan, N.; Khan, M.G.; Araci, S.; Mashwani, W.K.; Khan, B. Coefficient Estimates for a Subclass of Meromorphic Multivalent q-Close-to-Convex Functions. Symmetry 2021, 13, 1840. [CrossRef]

10. Srivastava, H.M.; Murugusundaramoorthy, G.; Sivasubramanian, S. Hypergeometric functions in the parabolic starlike and uniformly convex domains. Integr. Transf. Spec. Func. 2007, 18, 511-520. [CrossRef]

11. Yin, W.; Yang, W.; Liu, H. A neural network scheme for recovering scattering obstacles with limited phaseless far-field data. J. Comput. Phys. 2020, 417, 1-26. [CrossRef]

12. Yin, W.; Ge, J.; Meng, P. A neural network method for the inverse scattering problem of impenetrable cavities. Electron Res. Arch 2020, 28, 1123-1142. [CrossRef]

13. Cao, X.; Diao, H.; Liu, H.; Zou, J. On nodal and generalized singular structures of Laplacian eigen functions and applications to inverse scattering problems. J. Math. Pures Appl. 2020, 143, 116-161. [CrossRef]

14. Fang, X.; Deng, Y. Uniqueness on recovery of piecewise constant conductivity and inner core with one measurement. Inverse Probl. Imaging 2018, 12, 733-743. [CrossRef]

15. Blasten, E.; Liu, H. Scattering by curvatures, radiationless sources, transmission eigenfunctions, and inverse scattering problems. SIAM J. Math. Anal. 2021, 53, 3801-3837. [CrossRef]

16. Khan, M.G.; Ahmad, B.; Khan, N.; Mashwani, W.K.; Arjika, S.; Khan, B.; Chinram, R. Applications of Mittag-Leffler type poisson distribution to a subclass of analytic functions involving conic-type regions. J. Funct. Spaces 2021, 2021, 4343163.

17. Srivastava, H.M.; El-Deeb, S.M. Fuzzy differential subordinations based upon the Mittag-Leffler type Borel distribution. Symmetry 2021, 13, 1023. [CrossRef]

18. Srivastava, H.M. ; Murugusundaramoorty, G.; El-Deeb, S.M. Faber polynomial coefficient estimates of bi-close-to-convex functions connected with Borel distribution of the Mittag-Leffler-type. J. Nonlinear Var. Anal. 2021, 5, 103-118.

19. El-Deeb, S.M.; Murugusundaramoorty, G.; Alburaikan, A. A bi-Bazilevič functions based on the Mittag-Leffler-Type Borel distribution associated with Legendre polynomials. J. Math. Comput. Sci. 2021, 24, 235-245. [CrossRef]

20. Keogh, F.R.; Merkes, E.P. A coefficient inequality for certain classes of analytic functions. Proc. Am. Math. Soc. 1969, 20, 8-12. [CrossRef]

21. Ma, W.; Minda, D. A unified treatment of some special classes of univalent functions. In Proceedings of the Conference on Complex Analysis, Tianjin, China, 19-23 June 1992, Li, Z., Ren, F., Yang, L., Zhang, S.; Eds.; International Press: Cambridge, MA, USA, 1994; pp. 157-169.

22. Silverman, H. Partial sums of starlike and convex functions. J. Math. Anal. Appl. 1997, 209, 221-227. [CrossRef]

23. Khan, M.G.; Darus, M.; Ahmad, B.; Murugusundaramoorty, G.; Khan, R.; Khan, N. Meromorphic starlike functions with respect to symmetric points. Int. J. Anal. Appl. 2020, 18, 1037-1047.

24. Khan, M.G.; Ahmad, B.; Salleh, Z. On subclasses of harmonic mappings involving Frasin operator. Afr. Mat. 2021, 32, $1159-1171$. [CrossRef] 